\title{
Periconceptional Maternal Alcohol Consumption and Neural Tube Defects
}

\author{
Jennifer A. Makelarski ${ }^{1}$, Paul A. Romitti ${ }^{1,}{ }^{*}$, Lixian Sun ${ }^{1}$, Trudy L. Burns ${ }^{1}$, Charlotte M. \\ Druschel $^{2}$, Lucina Suarez ${ }^{3}$, Andrew F. Olshan ${ }^{4}$, Anna Maria Siega-Riz ${ }^{4}$, Richard S. OIney ${ }^{5}$, \\ and the National Birth Defects Prevention Study \\ ${ }^{1}$ Department of Epidemiology, The University of lowa, lowa City, lowa 52242 \\ ${ }^{2}$ New York State Department of Health, Congenital Malformations Registry, Troy, New York \\ 12180 \\ ${ }^{3}$ Texas Department of State Health Services, Environmental Epidemiology and Disease \\ Registries Section, Austin, Texas 78756 \\ ${ }^{4}$ Department of Epidemiology, Gillings School of Global Public Health, University of North \\ Carolina, Chapel Hill, North Carolina 27599 \\ ${ }^{5}$ Division of Birth Defects and Developmental Disabilities, National Center on Birth Defects and \\ Developmental Disabilities, Centers for Disease Control and Prevention, Atlanta, Georgia
}

\section{Abstract}

BACKGROUND—Neural tube defects (NTD)s, which occur when the neural tube fails to close during early gestation, are some of the most common birth defects worldwide. Alcohol is a known teratogen and has been shown to induce NTDs in animal studies, although most human studies have failed to corroborate these results. Using data from the National Birth Defects Prevention Study, associations between maternal reports of periconceptional (1 month prior through 2 months postconception) alcohol consumption and NTDs were examined.

METHODS—NTD cases and unaffected live born control infants, delivered from 1997 through 2005, were included. Interview reports of alcohol consumption (quantity, frequency, variability, and type) were obtained from 1223 case mothers and 6807 control mothers. Adjusted odds ratios (aOR)s and $95 \%$ confidence intervals were estimated using multivariable logistic regression analysis.

RESULTS-For all NTDs combined, most aORs for any alcohol consumption, one or more binge episodes, and different type(s) of alcohol consumed were near unity or modestly reduced $(叉 0.7<\mathrm{aOR} \leq 1.1)$ and were not statistically significant. Findings were similar for individual NTD subtypes.

CONCLUSIONS-These findings suggest no elevated association between maternal periconceptional alcohol consumption and NTDs. Underreporting of alcohol consumption, due to 
negative social stigma associated with alcohol consumption during pregnancy, and limited reports for mothers with early pregnancy loss of a fetus with an NTD may have affected the estimated odds ratios. Future studies should aim to increase sample sizes for less prevalent subtypes, reduce exposure misclassification, and improve ascertainment of fetal deaths and elective terminations.

\section{Keywords}

alcohol; birth defects; neural tube defects; pregnancy

\section{INTRODUCTION}

Neural tube defects (NTD)s are some of the most common birth defects worldwide. In the United States (US), more than 3000 NTD-affected pregnancies occur annually (Centers for Disease Control and Prevention, 2004). Survival of infants with NTDs varies by subtype. Anencephaly is invariably fatal, whereas children with spina bifida frequently experience severe disability (Date et al., 1993) and require continued medical treatment (Zurmohle et al., 1998). In 2003 dollars, Grosse et al. (2008) estimated the direct lifetime costs of spina bifida to be $\$ 560,000$ per child. Randomized controlled trials showed that folic acid supplementation could prevent at least one-half of all NTDs (Medical Research Council, 1991; Czeizel and Dudas, 1992), leading to mandatory folic acid fortification of enriched grains in the United States in 1998. Although NTD rates in the US decreased by an estimated 26\% following folic acid fortification (Centers for Disease Control and Prevention, 2004), persistence of NTDs suggests presence of other risk factors or that such fortification is not sufficient (Cavalli and Copp, 2002).

Alcohol is a known teratogen, with effects that may be more pronounced during organogenesis (Yanaguita et al., 2008). A potential association between alcohol consumption and NTDs was first described in a case series more than 25 years ago (Friedman, 1982); however, the biological mechanisms by which alcohol impacts neural tube development are not well understood. Animal models suggest that prenatal alcohol exposure in early development can lead to excessive neural crest cell death (Bannigan and Burke, 1982), as well as contribute to folic acid deficiency via increased excretion of folic acid by the kidneys (McMartin, 1984; Muldoon and McMartin, 1994).

Published human studies of maternal alcohol consumption and NTDs have produced results inconsistent with animal studies. Two studies (Mills and Graubard, 1987; McDonald et al., 1992), which did not control for folic acid intake, found no association between alcohol consumption during the first trimester of pregnancy and NTDs. Among studies that controlled for folic acid supplementation, three reported no association with any level of alcohol consumption examined (Shaw et al., 1996, 2002; Suarez et al., 2008), and one reported a significantly positive association [OR: 2.1 ; 95\% confidence interval (CI): 1.1, 4.0] with alcohol consumption of least once per week (Grewal et al., 2008).

Important limitations of previous epidemiologic studies were the lack of comprehensive analyses of important covariables and investigation of associations for NTD subtypes. Specifically, no study examined associations by type of alcohol consumed. Folate levels are thought to vary by type of alcohol. Larroque et al. (1992) reported a positive correlation 
between red cell folate levels and alcohol consumption in pregnant women, most of whom consumed beer. Stark et al. (2005) found similar results in pregnant African American women, with a non-significant, but positive association between beer consumption and plasma 5-MTHFA (the most common folate metabolite) and a significant negative association between wine cooler consumption and plasma 5-MTHFA. Also, due to limited data and/or small sample sizes, few previous studies were able to evaluate relevant covariables, including those known to be associated with NTDs (e.g., diabetes, exposure to folate antagonists) (Lammer et al., 1987; Becerra et al., 1990). Additionally, only two studies (Mills and Graubard, 1987; Grewal et al., 2008) examined NTD subtypes. This is important because of the potential developmental and etiologic heterogeneity in NTD development (Reviewed by Mitchell, 2005). The inability to stratify by NTD subtype could dilute reported associations due to this underlying heterogeneity.

Data from the National Birth Defects Prevention Study (NBDPS), an ongoing, populationbased, multisite case-control study, were used to investigate the association between maternal reports of alcohol consumption and NTD subtype. The NBDPS sample is sufficiently large to address existing limitations in the alcohol-NTD literature such as analysis by select subtypes, stratification by type of alcohol consumed, and examination of important covariables.

\section{MATERIALS AND METHODS}

The NBDPS was designed to investigate genetic and non-inherited risk factors for over 30 major, structural birth defects including NTD subtypes (British Pediatric Association, 1979]), anencephaly and craniorachischisis (740.000-702.100), spina bifida (741.000741.990), encephalocele, cranial meningocele, encephalomyelocele, and other rare subtypes (742.000-742.990). Case definitions for each eligible defect required confirmatory diagnostic procedures. The NBDPS excluded cases with known single gene or chromosomal etiologies. Clinical geneticists at each NBDPS site reviewed reports from medical records to determine case eligibility. A brief description of NBDPS methods is provided below; additional detail is reported elsewhere (Yoon et al., 2001; Rasmussen et al., 2001, 2003). Each site obtained institutional review board approval for the NBDPS and all participants provided informed consent.

Data included in this report were collected at 10 sites (Arkansas, California, Iowa, Massachusetts, New Jersey, New York, North Carolina, Texas, Utah, and metropolitan Atlanta), which cover an annual birth population of 482,000 , or about $10 \%$ of births nationally (Yoon et al., 2001). For this analysis, eligible NTD cases were live births (all sites), fetal deaths at 20 weeks or greater gestation (six sites), and elective terminations (five sites) with estimated dates of delivery (EDD)s from October 1, 1997 to December 31, 2005 and diagnosed with at least one NTD subtype (anencephaly, craniorachischisis, spina bifida, encephalocele, cranial meningocele, or encephalomyelocele). NTD cases were further classified as isolated (no additional major defects) or multiple (more than one major unrelated defect) phenotypes. Eligible control infants were live births without a structural birth defect delivered during the same time period and randomly selected using birth certificates or hospital records from the same regions as the NTD cases. 
Participating mothers of eligible NTD cases and control infants completed the NBDPS interview no earlier than 6 weeks and no later than 24 months following the EDDs. The median time between the EDDs and the completed interviews was 9.5 months for case and 8.9 months for control mothers. The NBDPS interview asked about maternal exposures, including infectious, chemical, physical, nutritional, and behavioral factors. For this study, the periconceptional period included the month before conception (B1) and the two months postconception (P1, P2). Including exposure reported in B1 allowed for analysis of mothers with unrecognized pregnancies who might have extended pre-pregnancy exposure patterns into P1 or later. The months, P1 and P2, encompass the relevant gestational period for development of NTDs.

\section{Alcohol Consumption}

The NBDPS interview collected data on quantity, frequency, and variability of alcohol consumption and on type(s) of alcohol consumed. Maternal periconceptional alcohol consumption was assessed using a previously developed approach (for details see Romitti et al., 2007). Mothers who reported consumption of alcohol during the periconceptional period were queried about the month(s) during which they drank (yes/no), the average number of drinking days per month (frequency), the average number of drinks per drinking day (quantity), the maximum number of drinks on one occasion per drinking month (variability), and type(s) of alcohol consumed (beer, wine, and/or distilled spirits).

Mothers were classified as exposed if they reported drinking alcohol during one or more periconceptional months. Maternal reports were excluded from analyses if, for any pregnancy month or trimester, alcohol consumption (yes/no) was missing or unknown or if reports of average drinking for any month were greater than 150 drinks. Alcohol consumption was also categorized by quantity-frequency reported. The average number of drinks per each drinking month was calculated by multiplying the reported average number of drinking days per month by the reported average number of drinks per drinking day for that month. A periconceptional average number of drinks per month (total average number of drinks per month divided by the number of months drank) and maximum average number of drinks per month (highest reported average number of drinks per month) were calculated for each mother. Using a 30-day month, four categories of consumption were used to classify the periconceptional average number of drinks per month and maximum average number of drinks per month: monthly to weekly (1-4 drinks per month); weekly to every other day (5-15 drinks per month); every other day to daily (16-30 drinks per month); and daily with more than one drink per day ( $>30$ drinks per month). Binge drinking, measured using both sex-neutral (Naimi et al., 2003) and sex-specific (Wechsler et al., 1995) norms, was also categorized. Sex-neutral norms included five or more drinks per day on average, on one occasion, or both, whereas sex-specific norms for females included four or more drinks per day on average, on one occasion, or both. Case and control mothers were classified into one of three categories: no consumption, consumption without binge drinking, or at least one binge drinking episode. Finally, mothers were classified by type(s) of alcohol consumed, including beer only, beer plus other alcohol type (wine and/or distilled spirits), or other alcohol type only. 


\section{Covariables}

Covariables evaluated included maternal age at delivery $(<21,21-25,26-30,31-35$, and $>35$ ), race/ethnicity (non-Hispanic white, non-Hispanic black, Hispanic, Other), education ( $<12$ years, 12 years, $13-15$ years, 16 or more years), gravidity $(0,1,2,3$, or more), prepregnancy body-mass index $(<18.5,18.5-24.9,25-29.9, \geq 30)$, periconceptional smoking (yes/no), and NBDPS site. Additionally, dietary folate equivalent intake ( $<600 \mu \mathrm{g}, \varangle 600 \mu \mathrm{g})$ and periconceptional use of folic-acid-containing supplements (yes/no) were examined. Dietary folate intake was assessed using responses to the Willet Food Frequency questionnaire (Willett et al., 1985, 1987) adapted for the NBDPS interview and measured food intake during the one year before conception and from reports of breakfast cereals consumed during P1 and P2. Dietary folate equivalents [DFE]s were estimated using the reported food frequencies, the standardized serving size on which a question item was based, and the United States Department of Agriculture National Standard Reference 16-1 (United States Department of Agriculture, 2004). Also, the NBDPS interview queried mothers regarding their intake of vitamins and supplements for a period of 3 months before conception through delivery. For each supplement reported, mothers were asked to provide start and stop dates (or if dates were unknown, duration of use) and frequency of intake. Each supplement was assessed to determine whether or not it contained folic acid. Eligible mothers were classified into two groups, those who took folic-acid-containing supplements during the periconceptional period and those who did not.

\section{Statistical Analysis}

Analyses were conducted using SAS software, version 9.2 (SAS Institute Inc, 2007). Descriptive analyses of NTD case and maternal characteristics were conducted by NTD subtype and compared to control infants and mothers using chi-square tests. Crude odds ratios (OR)s and 95\% CIs were estimated to examine associations between any maternal periconceptional alcohol consumption, average and maximum average drinks per month, binge episodes, and alcohol type and all NTD cases combined. Results from descriptive analyses were used to construct the most parsimonious multivariable logistic regression models to predict NTDs using backward selection approaches. Covariables included in the preliminary model were those which, based on the descriptive analysis, were associated ( $p<$ 0.20 ) with any alcohol consumption (yes/no) and/or NTD outcome; maternal age, BMI, and dietary food folate were entered as continuous variables. Backward selection was used to exclude covariables from the preliminary model beginning with the least statistically significant covariable (highest $p$-value) based on the Wald chi-square statistic. As each covariable was removed, the fit of the full model was compared to the reduced model fit using the log-likelihood ratio (LLR) test. Covariables for which the LLR was significant ( $p$ $<0.05$ ), were re-entered into the model. Also, regardless of LLR values, covariables for which exclusion from the model resulted in a change in parameter estimate of an alcohol exposure variable by greater than $20 \%$ were reentered in the model. Based on the final multivariable logistic model, adjusted odds ratios (aORs) were estimated to characterize the association between all NTD cases combined and any periconceptional alcohol consumption, quantity-frequency of consumption, binge episodes, and type of alcohol consumed. In addition, aORs for maximum average monthly drinks and binge drinking were 
stratified by type of alcohol, folic acid consumption, pregnancy intendedness (planned versus unplanned pregnancy) and family history of a NTD; aORs for type of alcohol were stratified by folic acid consumption. Finally, all analyses were conducted restricting infants to those sites who collected information on live births, fetal deaths, and elective terminations (five sites) and restricted to mothers exposed in P1 with/without exposure in P2 only. When numbers were sufficient, these subanalyses were also conducted by NTD subtype and phenotype.

\section{RESULTS}

Interview data were collected from mothers of 1223 (68\% of eligible) NTD cases and 6807 ( $66 \%$ of eligible) control infants. Of these, 56 case and 204 control mother interviews were excluded due to: incomplete interviews ( case $=17$; control $=104)$; maternal diagnosis of type 1 or type 2 diabetes before or during the index pregnancy (case $=18$; control $=42$ ); and maternal periconceptional exposure to known folic acid antagonists (case $=21$; control $=$ 58). To improve homogeneity of NTD subtype groups, maternal interviews for an additional seven NTD cases were excluded due to diagnosis of multiple NTD subtypes for each case. Among the 1160 NTD cases included in the analyses, 328 were diagnosed with anencephaly or craniorachischisis, 703 with spina bifida, and 129 with another rare subtype.

Compared to control mothers, case mothers (all NTDs combined) were significantly more likely to be Hispanic, less educated, and to differ in proportions by site (Table 1). Anencephaly cases were more likely to be female and preterm ( $<37$ weeks gestation) compared to control infants. Mothers of anencephaly cases were less likely to have reported periconceptional smoking, whereas those of spina bifida cases tended to be younger and have a pre-pregnancy body-mass index of 30 or more. Mothers of spina bifida and anencephaly cases were each more likely to have had four or more pregnancies than control mothers. Case and control mothers did not differ in use of folic-acid-containing supplements or food folate consumption.

Thirty percent of case mothers and approximately $36 \%$ of control mothers reported periconceptional alcohol consumption (Table 2); pattern of use was similar between the two groups. Case mothers were more likely to report consumption of beer only, whereas control mothers were more likely to report consumption of other alcohol types. Duration and type of alcohol consumed were similar among mothers of infants of each NTD subtype. When stratified by 6-month intervals between EDD and time to interview (1-6 months, 7-12 months, 13-18 months, and 19-24 months), consumption frequency between case and control mothers was similar as the time to interview increased; however, in control mothers, the frequency was somewhat lower for those with the shortest (1-6 months) and longest (18-24 months) intervals, 38 and 25\%, respectively (data not shown).

After adjustment for maternal race/ethnicity, education, pre-pregnancy body-mass index, periconceptional smoking, and NBDPS site, any maternal periconceptional alcohol consumption was not associated with all NTD cases combined. Similarly, when compared to no periconceptional alcohol consumption, no associations were found for maximum average monthly drinks (Table 3) or sex-specific binge episodes (Table 4) for all NTD cases 
combined. Also, no patterns were seen with increasing number of maximum average drinks. Results for binge episodes did not appreciably change when using sex-neutral norms (data not shown). Adjusted analyses for all NTD cases combined for reported type(s) of alcohol consumed also did not show a pattern of elevated odds ratios (Table 5). Results were similar for each subtype and for isolated NTD cases only.

The aORs for maximum number of monthly drinks and one or more binge episodes did not change appreciably when stratified by type of alcohol consumed, folic acid supplementation, pregnancy intendedness, or family history of an NTD; however, odds were significantly elevated in mothers of spina bifida cases who reported no folic acid consumption and beer consumption only compared to no alcohol consumption ( $\mathrm{aOR}=1.8, \mathrm{CI}=1.1-2.9)$. Further, sub-analyses restricted to the five sites which ascertained live births, fetal deaths ( 220 weeks gestation), and elective terminations produced little change in the aORs (data not shown). Sub-analyses restricted to mothers exposed in P1 with/without exposure in $\mathrm{P} 2$ only produced similar results.

\section{DISCUSSION}

The current study included nearly twice as many NTD cases as the largest prior study (Shaw et al., 1996) and improved upon exposure classification, allowing for sub-analyses by alcohol type. Even with these design enhancements, the current study did not identify a pattern of elevated associations between any maternal periconceptional alcohol consumption and all NTD cases combined or any NTD subtype. It also did not identify elevated associations for reported maximum average monthly drinks for all NTD cases combined and most NTD subtypes. Additionally, little variation in the magnitude of associations by type(s) of alcohol consumed was found. Despite the relatively large sample size, the relatively low number of NTDs, particularly in subtype analyses, resulted in imprecise estimates.

The analyses of maternal periconceptional alcohol consumption and NTDs used data from one of the largest US population-based, case-control studies of birth defects. With our sample size and a $36 \%$ rate of periconceptional alcohol consumption in controls, a priori power calculations showed that our minimum detectable odds ratio for an association between any periconceptional alcohol consumption (yes/no) and NTDs combined was 1.15. The results were similar to the six previously published studies identified that examined the association between maternal alcohol consumption and NTDs (Mills and Graubard, 1987; McDonald et al., 1992; Shaw et al., 1996, 2002; Suarez et al., 2008). Among these studies, only one study (Grewal et al., 2008) reported a significant, positive association (with $\geq 1$ drink per week). Notably, this positive association was found only in adjusted analyses. No prior study had examined the association between alcohol exposure and NTDs by type of alcohol consumed, although prior work suggested that beer consumption increased red cell folate and plasma 5-MTHFA (Larroque et al., 1992; Stark et al. 2005). Because of the wellestablished role of folate in preventing NTDs (Medical Research Council, 1991; Czeizel and Dudas, 1992), this study examined the association between alcohol exposure and NTDs by alcohol type, but found no variation in risk. 
The current study improved upon prior studies in several ways. The primary strength of this analysis was the large, population-based, geographically and ethnically diverse sample. The use of a population-based sample reduced the risk of selection bias. Comparison of selected maternal characteristics of controls versus all live births at each site has shown that NBDPS participants tend to be similar to all live births (Cogswell et al., 2009). Also, all NTD cases were reviewed and verified by clinical geneticists, decreasing the risk of case misclassification. In addition, the large sample size allowed for stratification by NTD subtype, which is important due to the potential heterogeneity of risk factors among subtypes.

Exposure data collection was conducted through detailed maternal interview reports using a structured questionnaire. Detailed data allowed assessment of alcohol consumption by quantity, frequency, and variability, as well as alcohol type, which had not previously been investigated. With the use of retrospective reports, the potential for differential recall existed between case and control mothers, although Verkerk et al. (1994) reported no significant differences in prospective and retrospective reports of alcohol between case and control mothers. Also, in the current study, the frequency of reported periconceptional alcohol consumption for case and control mothers stratified by 6-month intervals were similar as the time to interview increased.

Despite improvements upon prior similar studies, the lack of a positive association may be explained in several ways. There may in fact be no positive association between maternal periconceptional alcohol exposure and NTDs, although this conclusion is inconsistent with previous animal studies (Graham and Ferm, 1985; Hunter et al., 1994; Chen et al., 2005; Yanaguita et al., 2008). As mentioned, the biological mechanisms by which alcohol impacts neural tube development are not well understood. Animal models suggest that prenatal alcohol exposure in early development leads to excessive cell death (Bannigan and Burke, 1982), particularly among premigratory neural crest cells (Kotch and Sulik, 1992). This may result in too few cells for fusion of the neural folds (Copp et al., 2003; Greene and Kopp, 2006). Animal models also suggest that alcohol exposure may play an indirect role in NTD development (Yanaguita et al., 2008) by contributing to folic acid deficiency via increased excretion of folic acid by the kidneys (McMartin, 1984; Muldoon and McMartin, 1994).

The discrepant findings between animal and human studies may be explained, in part, by methodological limitations of human studies. For example, misclassification of timing and dose of alcohol could bias results. In the NBDPS, mothers were not queried regarding the precise volume of alcohol drinks consumed, rather general volumes (one can of beer, one glass of wine, and one shot of liquor) were assumed. Varying alcohol concentrations between types of alcohol could have decreased differences in odds ratios by type(s) of alcohol consumed. Also, alcohol consumption and dose were self-reported retrospectively, and may have been under-reported due to the negative social stigma associated with alcohol consumption during pregnancy, especially when the health of the infant is known. It is unclear if this would result in non-differential or differential exposure misclassification and the impact on the magnitude and direction of the odds ratios is uncertain. An alternative explanation for the discrepant results with animal studies is that the lack of association seen here may be due to selective early pregnancy loss of fetuses with NTDs creating an 
ascertainment/survival bias; a large proportion of pregnancies affected by NTDs result in early fetal deaths (e.g., <20 weeks) (Centers for Disease Control and Prevention, 2004). Such cases are difficult to include in retrospective case-control studies, as the pregnancy may not have been recognized or the defect may not have been identified at the time of loss. Alcohol consumption has been associated with early pregnancy loss (Sokol, 1980; Henriksen et al., 2004) and could increase the likelihood of such study bias (Khoury et al., 1992).

In summary, the association between periconceptional alcohol consumption and NTDs was investigated using a large, case-control study. Results suggested no pattern of increasing odds per amount of alcohol consumed. Although these findings corroborate the null findings of previous studies, and efforts were made to improve study design, these results should be interpreted cautiously, as a few limitations exist. Future studies should aim to increase sample sizes for less prevalent subtypes, reduce exposure misclassification, and improve ascertainment of fetal deaths and elective terminations.

\section{Acknowledgments}

Supported by a grant from the Centers for Disease Control and Prevention (5U50DD000317 and 1U50DD000730).

The findings and conclusions in this report are those of the authors and do not necessarily represent the official position of the Centers for Disease Control and Prevention.

\section{References}

Bannigan J, Burke P. Ethanol teratogenicity in mice: a light microscopic study. Teratology. 1982; 26:247-254. [PubMed: 7163973]

Becerra JE, Khoury MJ, Cordero JF, et al. Diabetes mellitus during pregnancy and the risks for specific birth defects: a population-based case-control study. Pediatrics. 1990; 85:1-9. [PubMed: 2404255]

British Paediatric Association. British Paediatric Association Classification of Diseases. London: 1979.

Cavalli P, Copp A. Inositol and folate resistant neural tube defects. J Med Genet. 2002; 39:e5. [PubMed: 11836374]

Centers for Disease Control and Prevention. Spina bifida and anencephaly before and after folic acid mandate-United States, 1995-1996 and 1999-2000. MMWR Morb Mortal Wkly Rep. 2004; 53:362-365. [PubMed: 15129193]

Chen SY, Charness ME, Wilkemeyer MF, et al. Peptide-mediated protection from ethanol-induced neural tube defects. Dev Neurosci. 2005; 27:13-19. [PubMed: 15886480]

Cogswell ME, Bitsko RH, Anderka M, et al. Control selection and participation in an ongoing, population-based, case-control study of birth defects: the National Birth Defects Prevention Study. Am J Epidemiol. 2009; 170:975-985. [PubMed: 19736223]

Copp A, Greene N, Murdoch J. The genetic basis of mammalian neurulation. Nature. 2003; 4:784793.

Czeizel AE, Dudas I. Prevention of the first occurrence of neural-tube defects by periconceptional vitamin supplementation. N Engl J Med. 1992; 327:1832-1835. [PubMed: 1307234]

Date I, Yagyu Y, Asari S, et al. Long-term outcome in surgically treated spina bifida cystica. Surg Neurol. 1993; 40:471-475. [PubMed: 8235969]

Friedman JM. Can maternal alcohol ingestion cause neural tube defects? J Pediatr. 1982; 101:232-234. [PubMed: 7097418] 
Graham JM Jr, Ferm VH. Heat- and alcohol-induced neural tube defects: interactions with folate in a golden hamster model. Pediatr Res. 1985; 19:247-251. [PubMed: 3982887]

Greene, N.; Copp, A. The embryonic basis of neural tube defects. In: Wyszynski, D., editor. Neural tube defects: From origin to treatment. New York: Oxford University Press; 2006. p. 15-28.

Grewal J, Carmichael SL, Ma C, et al. Maternal periconceptional smoking and alcohol consumption and risk for select congenital anomalies. Birth Defects Res A Clin Mol Teratol. 2008; 82:519-526. [PubMed: 18481814]

Grosse SD, Ouyang L, Collins JS, et al. Economic evaluation of a neural tube defect recurrenceprevention program. Am J Prev Med. 2008; 35:572-577. [PubMed: 18845415]

Henriksen TB, Hjollund NH, Jensen TK, et al. Alcohol consumption at the time of conception and spontaneous abortion. Am J Epidemiol. 2004; 160:661-667. [PubMed: 15383410]

Hunter ES, Tugman JA, Sulik KK, et al. Effects of short-term exposure to ethanol on mouse embryos in vitro. Toxicol In Vitro. 1994; 8:413-421. [PubMed: 20692933]

Khoury MJ, James LM, Flanders WD, et al. Interpretation of recurring weak associations obtained from epidemiologic studies of suspected human teratogens. Teratology. 1992; 46:69-77. [PubMed: 1641813]

Kotch LE, Sulik KK. Patterns of ethanol-induced cell death in the developing nervous system of mice; neural fold states through the time of anterior neural tube closure. Int J Dev Neurosci. 1992; 10:273-279. [PubMed: 1414440]

Lammer EJ, Sever LE, Oakley GP Jr. Teratogen update: valproic acid. Teratology. 1987; 35:465-473. [PubMed: 3114906]

Larroque B, Kaminski M, Lelong N, et al. Folate status during pregnancy: relationship with alcohol consumption, other maternal risk factors and pregnancy outcome. Eur J Obstet Gynecol Reprod Biol. 1992; 43:19-27. [PubMed: 1737604]

McDonald A, Armstrong B, Sloan M. Cigarette, alcohol, and coffee consumptions and congenital defects. Am J Public Health. 1992; 82:91-93. [PubMed: 1536342]

McMartin KE. Increased urinary folate excretion and decreased plasma folate levels in the rat after acute ethanol treatment. Alcohol Clin Exp Res. 1984; 8:172-178. [PubMed: 6375428]

Medical Research Council. Prevention of neural tube defects: results of the Medical Research Council Vitamin Study. MRC Vitamin Study Research Group. Lancet. 1991; 338:131-137. [PubMed: 1677062]

Mills JL, Graubard BI. Is moderate drinking during pregnancy associated with an increased risk for malformations? Pediatrics. 1987; 80:309-314. [PubMed: 3627880]

Mitchell LE. Epidemiology of neural tube defects. Am J Med Genet C Semin Med Genet. 2005; 135C: 88-94. [PubMed: 15800877]

Muldoon RT, McMartin KE. Ethanol acutely impairs the renal conservation of 5methyltetrahydrofolate in the isolated perfused rat kidney. Alcohol Clin Exp Res. 1994; 18:333339. [PubMed: 8048735]

Naimi TS, Brewer RD, Mokdad A, et al. Binge drinking among US adults. JAMA. 2003; 289:70-75. [PubMed: 12503979]

Rasmussen SA, Moore CA, Paulozzi LJ, et al. Risk for birth defects among premature infants: a population-based study. J Pediatr. 2001; 138:668-673. [PubMed: 11343041]

Rasmussen SA, Olney RS, Holmes LB, et al. Guidelines for case classification for the National Birth Defects Prevention Study. Birth Defects Res A Clin Mol Teratol. 2003; 67:193-201. [PubMed: 12797461]

Romitti PA, Sun L, Honein MA, et al. Maternal periconceptional alcohol consumption and risk of orofacial clefts. Am J Epidemiol. 2007; 166:775-785. [PubMed: 17609516]

Shaw GM, Nelson V, Carmichael SL, et al. Maternal periconceptional vitamins: interactions with selected factors and congenital anomalies? Epidemiology. 2002; 13:625-630. [PubMed: 12410002]

Shaw GM, Velie EM, Morland KB. Parental recreational drug use and risk for neural tube defects. Am J Epidemiol. 1996; 144:1155-1160. [PubMed: 8956628]

Sokol RJ. Alcohol and spontaneous abortion. Lancet. 1980; 316:1079. [PubMed: 6107699] 
Stark KD, Pawlosky RJ, Beblo S, et al. Status of plasma folate after folic acid fortification of the food supply in pregnant African American women and the influences of diet, smoking, and alcohol consumption. Am J Clin Nutr. 2005; 81:669-677. [PubMed: 15755838]

Suarez L, Felkner M, Brender JD, et al. Maternal exposures to cigarette smoke, alcohol, and street drugs and neural tube defect occurrence in offspring. Matern Child Health J. 2008; 12:394-401. [PubMed: 17641961]

US Department of Agriculture ARS, USDA Nutrient Data, \& Laboratory. USDA National Nutrient Database for Standard Reference, Release 16. 2004

Verkerk PH, Buitendijk SE, Verloove-Vanhorick SP. Differential misclassification of alcohol and cigarette consumption by pregnancy outcome. Int J Epidemiol. 1994; 23:1218-1225. [PubMed: 7721524]

Wechsler H, Dowdall GW, Davenport A, et al. A gender-specific measure of binge drinking among college students. Am J Public Health. 1995; 85:982-985. [PubMed: 7604925]

Willett WC, Reynolds RD, Cottrell-Hoehner S, et al. Validation of a semi-quantitative food frequency questionnaire: comparison with a 1-year diet record. J Am Diet Assoc. 1987; 87:43-47. [PubMed: 3794132]

Willett WC, Sampson L, Stampfer MJ, et al. Reproducibility and validity of a semiquantitative food frequency questionnaire. Am J Epidemiol. 1985; 122:51-65. [PubMed: 4014201]

Yanaguita MY, Gutierrez CM, Ribeiro CN, et al. Pregnancy outcome in ethanol-treated mice with folic acid supplementation in saccharose. Childs Nerv Syst. 2008; 24:99-104. [PubMed: 17619885]

Yoon PW, Rasmussen SA, Lynberg MC, et al. The National Birth Defects Prevention Study. Public Health Rep. 2001; 116(Suppl 1):32-40. [PubMed: 11889273]

Zurmohle UM, Homann T, Schroeter C, et al. Psychosocial adjustment of children with spina bifida. J Child Neurol. 1998; 13:64-70. [PubMed: 9512305] 


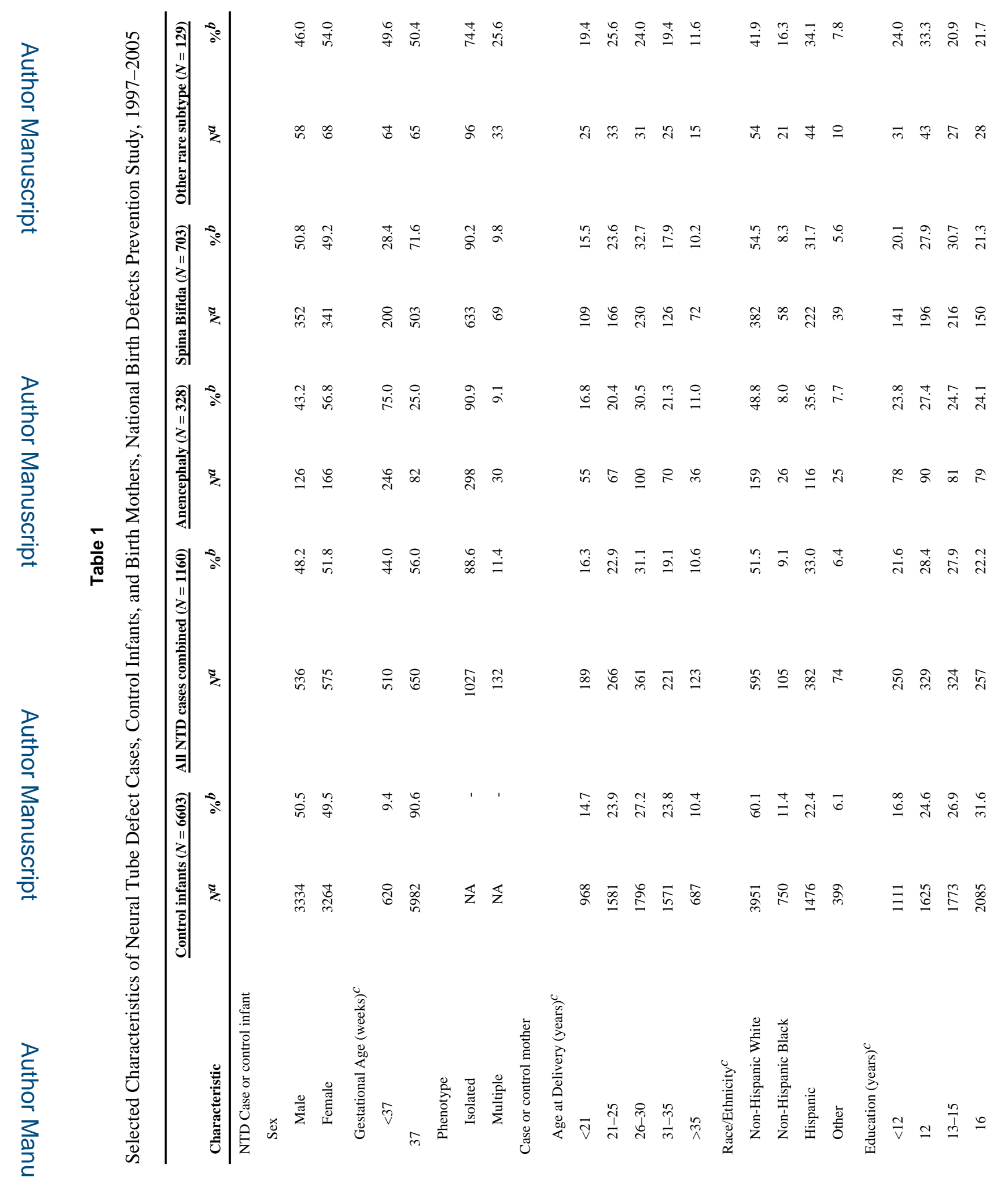

Birth Defects Res A Clin Mol Teratol. Author manuscript; available in PMC 2015 May 18. 


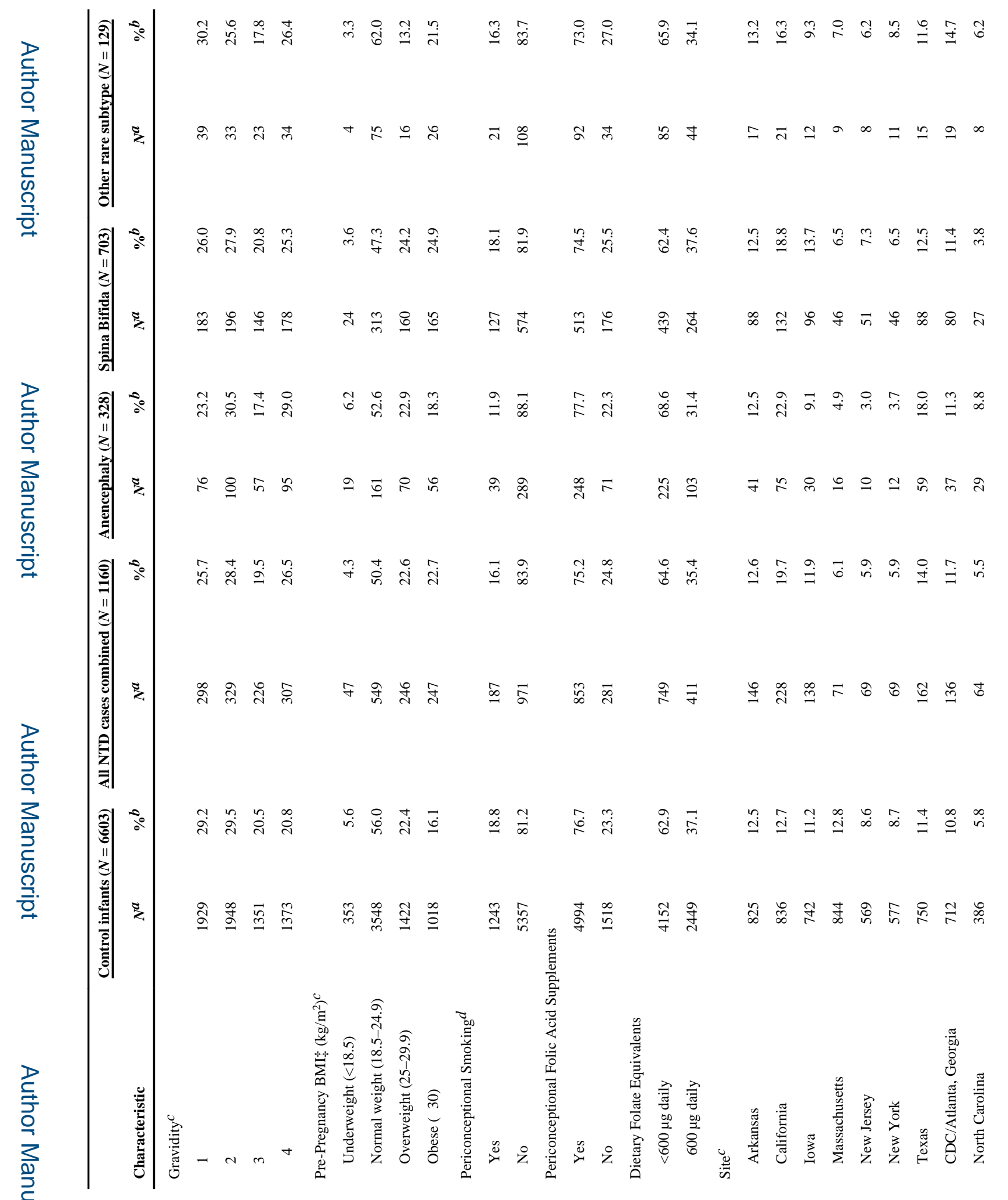

Birth Defects Res A Clin Mol Teratol. Author manuscript; available in PMC 2015 May 18. 


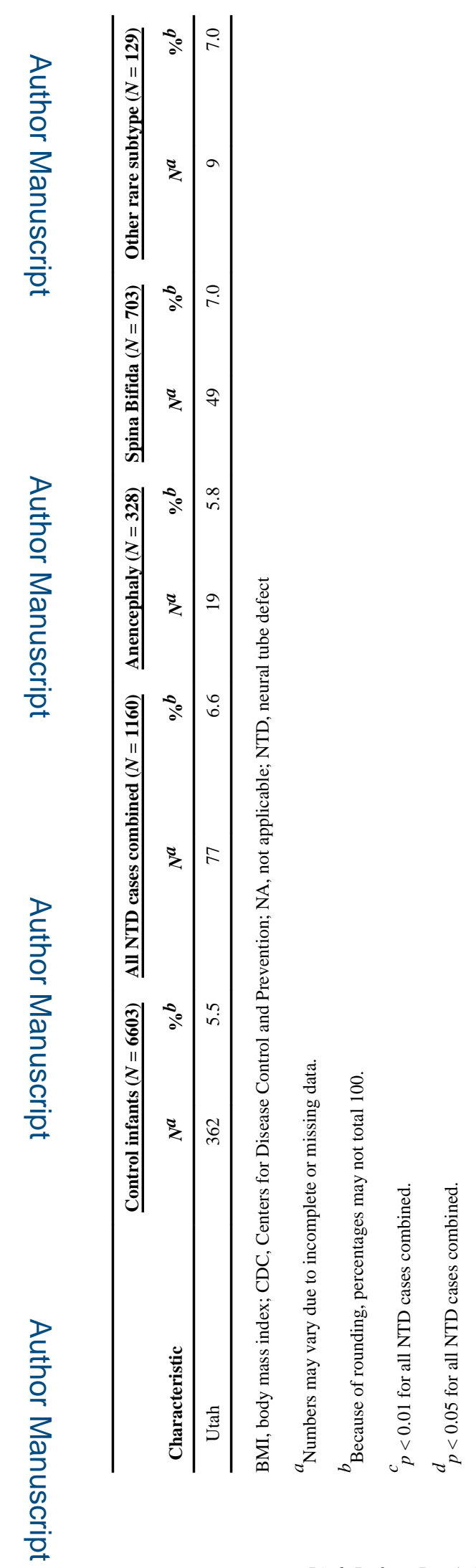

Birth Defects Res A Clin Mol Teratol. Author manuscript; available in PMC 2015 May 18. 


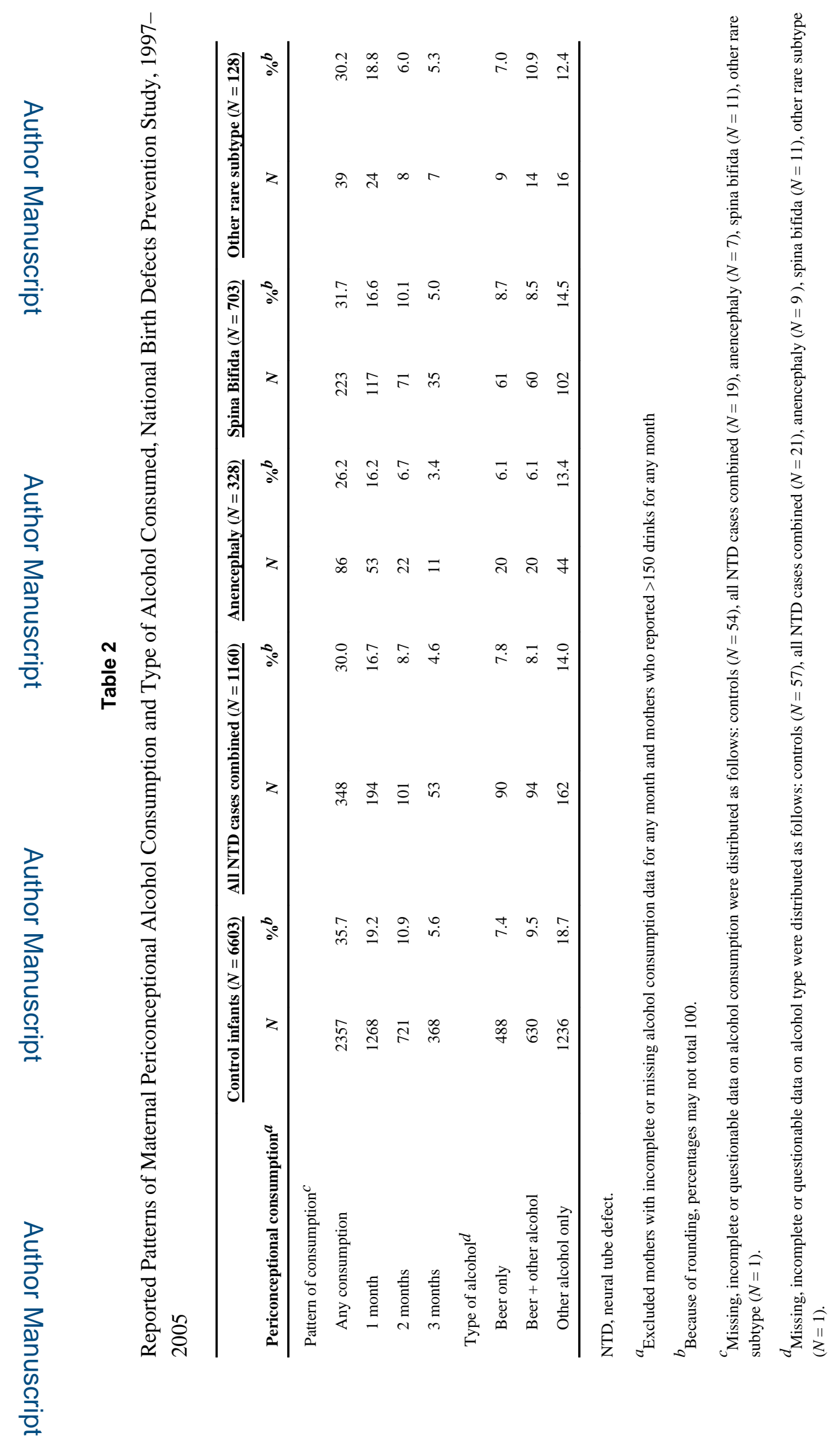

Birth Defects Res A Clin Mol Teratol. Author manuscript; available in PMC 2015 May 18. 
Makelarski et al.

Page 16

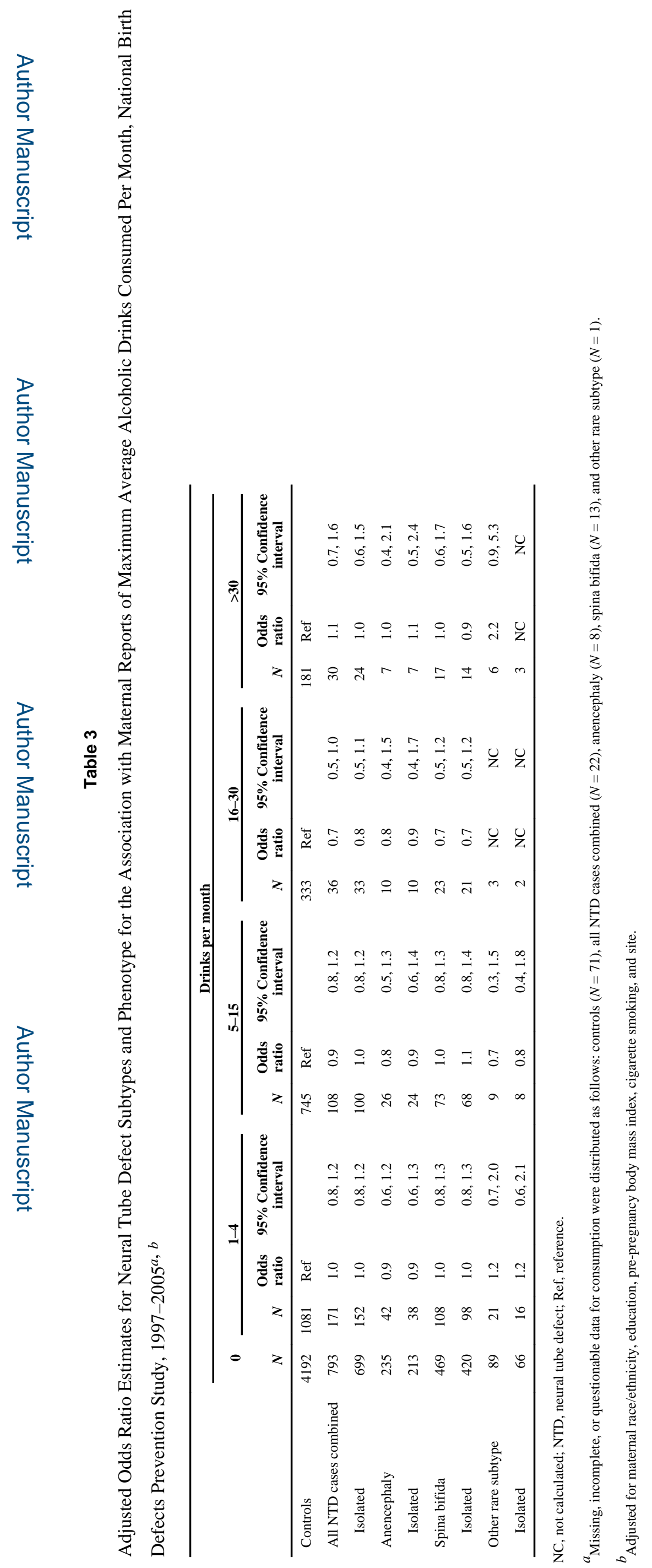

Birth Defects Res A Chin Mol Teratol. Author manuscript; available in PMC 2015 May 18. 


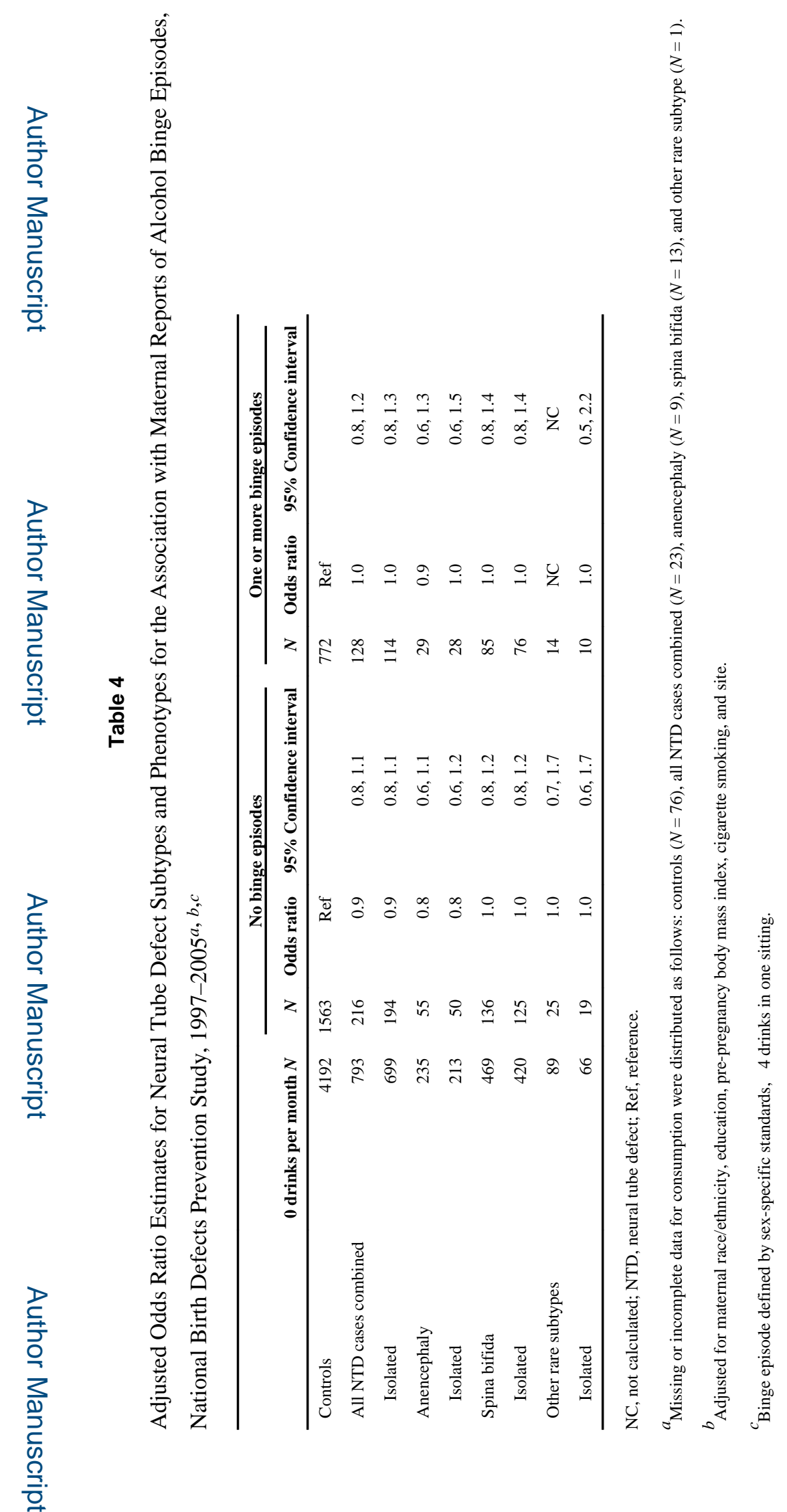

Birth Defects Res A Clin Mol Teratol. Author manuscript; available in PMC 2015 May 18. 


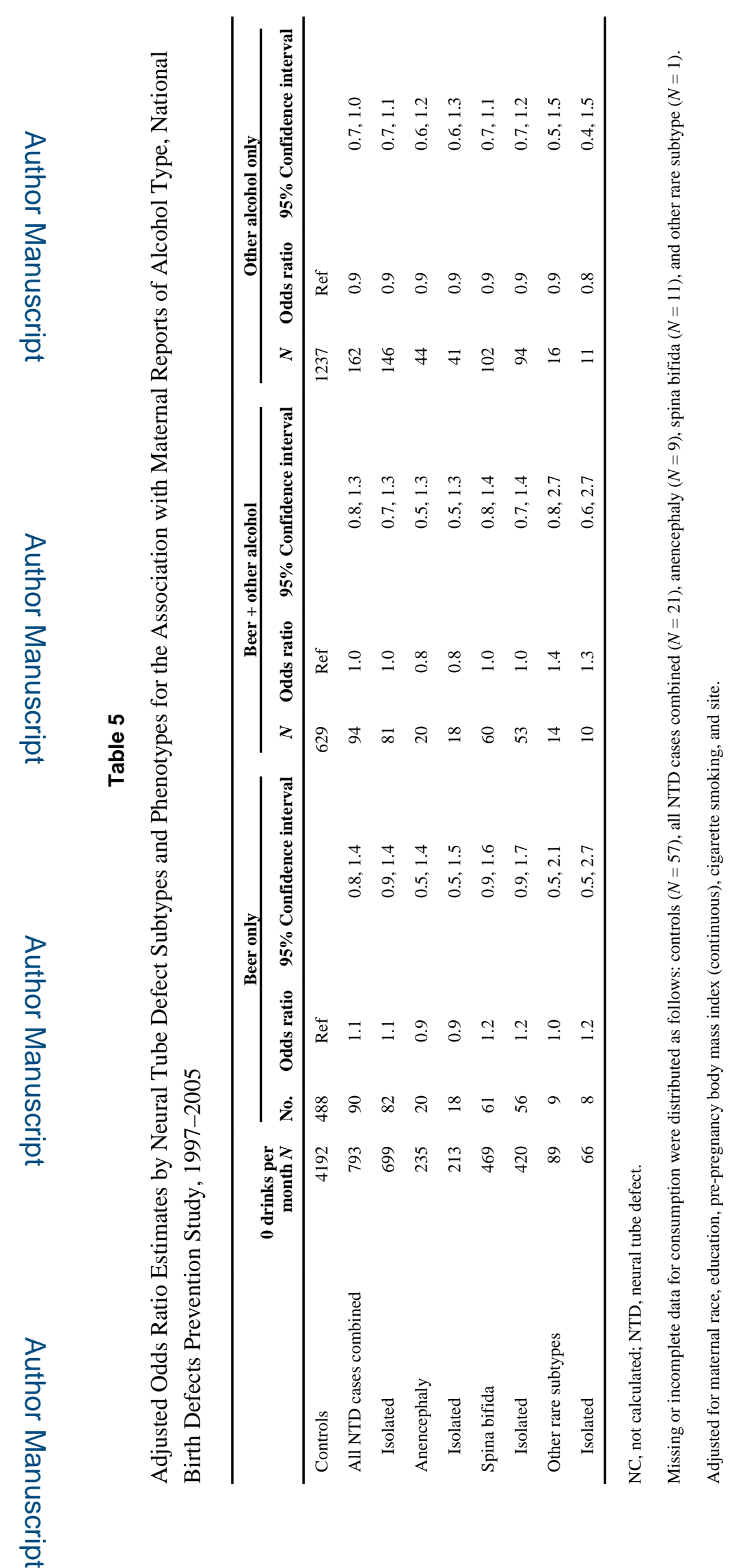

Birth Defects Res A Clin Mol Teratol. Author manuscript; available in PMC 2015 May 18. 